\title{
ON THE CONVERGENCE CLASSES FOR ENTIRE FUNCTIONS OF SEVERAL COMPLEX VARIABLES
}

O. M. Mulyava, M. M. Sheremeta. On the convergence classes for entire functions of several complex variables, Mat. Stud. 46 (2016), 67-71.

Results on the belonging of entire functions to generalized convergence classes earlier obtained by the authors are carried over on entire functions of several complex variables.

\section{Introduction. Let}

$$
f(z)=\sum_{k=0}^{\infty} a_{k} z^{k}
$$

be an entire transcendental function, $M(r, f)=\max \{|f(z)|:|z|=r\}$ and $\mu(r, f)=$ $=\max \left\{\left|a_{k}\right|: k \geq 0\right\}$ be the maximal term of the series (1). Let $L$ be class of continuous functions $\alpha$ such that $\alpha(x) \geq 0$ for $x \geq x_{0}, \alpha(x)=\alpha\left(x_{0}\right)$ for $x \leq x_{0}$ and on $\left[x_{0},+\infty\right)$ the function $\alpha$ increases to $+\infty$. We say that $\alpha \in L^{0}$ if $\alpha \in L$ and $\alpha(x(1+o(1)))=$ $=(1+o(1)) \alpha(x)$ as $x \rightarrow+\infty$ and remark that if $\alpha \in L^{0}$ then [1] $\varlimsup_{x \rightarrow+\infty} \frac{\alpha(\lambda x)}{\alpha(x)}=c(\lambda)<+\infty$ for each $\lambda \in[1,+\infty)$.

For $\alpha \in L$ and $\beta \in L$ we say that $f$ belongs to the generalized convergence class if

$$
\int_{r_{0}}^{\infty} \frac{\alpha(\ln M(r, f))}{r \beta(\ln r)} d r<+\infty
$$

We remark that $\mu(r, f) \leq M(r, f) \leq 2 \mu(2 r, f)$. Therefore, if $\alpha \in L^{0}$ and $\beta \in L^{0}$, then (2) holds iff

$$
\int_{r_{0}}^{\infty} \frac{\alpha(\ln \mu(r, f))}{r \beta(\ln r)} d r<+\infty .
$$

Theorem 1 from [2] for entire Dirichlet series implies the following statement.

Theorem A. Let $\alpha \in L$ be a concave function on $\left[x_{0},+\infty\right)$ and $\alpha\left(e^{x}\right) \in L^{0}$. Let a function $\beta \in L^{0}$ satisfy the condition $x \beta^{\prime}(x) / \beta(x) \geq h>0$ on $\left[x_{0},+\infty\right)$ and

$$
\int_{x_{0}}^{\infty} \frac{\alpha(x)}{\beta(x)} d x<+\infty
$$

2010 Mathematics Subject Classification: 30B50.

Keywords: entire function of several variables; convergence class. doi:10.15330/ms.46.1.67-71

(C) O. M. Mulyava, M. M. Sheremeta, 2016 
In order that $f$ belong to generalized convergence class it is necessary and in the case when $\left|a_{k}\right| /\left|a_{k+1}\right| \nearrow+\infty$ as $k_{0} \leq k \rightarrow \infty$ it is sufficient that

$$
\sum_{k=0}^{\infty}(\alpha(k)-\alpha(k-1)) \beta_{1}\left(\frac{1}{k} \ln \frac{1}{\left|a_{k}\right|}\right)<+\infty, \quad \beta_{1}(x)=\int_{x}^{\infty} \frac{d t}{\beta(t)} .
$$

By $\Omega$ we denote the class of positive functions $\Phi$ unbounded on $(-\infty,+\infty)$ such that the derivative $\Phi^{\prime}$ is continuously differentiable, positive and increasing to $+\infty$ on $(-\infty,+\infty)$ and we say that an entire function (1) belongs to the convergence $\Phi$-class if

$$
\int_{r_{0}}^{\infty} \frac{\Phi^{\prime}(\ln r) \ln M(r, f)}{\Phi^{2}(\ln r)} d \ln r<+\infty .
$$

In [3] it is proved that if $\Phi \in \Omega$ and

$$
\int_{r_{0}}^{\infty} \frac{\Phi^{\prime}(\sigma) \ln \Phi^{\prime}(\sigma)}{\Phi^{2}(\sigma)} d \sigma<+\infty
$$

then (6) holds iff

$$
\int_{r_{0}}^{\infty} \frac{\Phi^{\prime}(\ln r) \ln \mu(r, f)}{\Phi^{2}(\ln r)} d \ln r<+\infty .
$$

On the other hand, Theorem 1 from [4] implies the following statement.

Theorem B. Let $\Phi \in \Omega$ and

$$
0<h \leq \frac{\Phi^{\prime \prime}(\sigma) \Phi(\sigma)}{\left(\Phi^{\prime}(\sigma)\right)^{2}} \leq H<+\infty
$$

In order that (8) hold it is necessary and in the case when $\left|a_{k}\right| /\left|a_{k+1}\right| \nearrow+\infty$ as $k_{0} \leq k \rightarrow \infty$ it is sufficient that

$$
\sum_{k=0}^{\infty} \frac{1}{\Phi^{\prime}\left(\frac{1}{k} \ln \frac{1}{\left|a_{k}\right|}\right)}<+\infty
$$

Clearly, by condition (9) from Theorem B we get the necessary and sufficient condition for belonging of $f$ to the convergence $\Phi$-class.

Here we carry over these results on entire functions of several complex variables.

We remark that in [7] it is investigated the belonging to convergence classes of the functions represented by series, similar to the Taylor-Dirichlet series.

2. Generalized convergence class. Let $Z=\left(z_{1}, \ldots, z_{n}\right) \in \mathbb{C}^{n}$. We consider the exhaustion of the space $\mathbb{C}^{n}$ by the system $\left(G_{R}\right)_{R \geq 0}$ of a complete multiple-circular domains with the center at the point $(0, \ldots, 0) \in \mathbb{C}^{n}$. We assume that this system satisfies the conditions:

i) $\bigcup_{R \geq 0} G_{R}=\mathbb{C}^{n}$

ii) $\left(\forall R_{1}<R_{2}\right): G_{R_{1}} \subset G_{R_{2}}$;

iii) $\left(z_{1}, \ldots, z_{n}\right) \in G_{1} \Longleftrightarrow(\forall R>0):\left(R z_{1}, \ldots, R z_{n}\right) \in G_{R}$;

iv) $\left(z_{1}, \ldots, z_{n}\right) \in G_{R} \Longrightarrow\left(\forall\left(\theta_{1}, \ldots, \theta_{n}\right) \in \mathbb{R}^{n}\right):\left(z_{1} e^{i \theta_{1}}, \ldots, z_{n} e^{i \theta_{n}}\right) \in G_{R}$.

We denote $d_{\bar{G}}\left(k_{1}, \ldots, k_{n}\right)=\max \left\{|z|_{1}^{k_{1}} \ldots|z|_{n}^{k_{n}}: Z \in \bar{G}=\overline{G_{1}}\right\}$ and suppose that if $Z=\left(z_{1}, z_{1}, \ldots, z_{n}\right) \in \bar{G}$ then $\left|z_{j}\right| \geq l>0$ for all $j=1, \ldots, n$. 
Let

$$
f(Z)=f\left(z_{1}, z_{1}, \ldots, z_{n}\right)=\sum_{k_{1} \ldots k_{n}=0}^{\infty} a_{k_{1}, \ldots, k_{n}} z_{1}^{k_{1}} \ldots z_{n}^{k_{n}}
$$

be an entire transcendental function. We put $M_{\bar{G}}(R)=M_{\bar{G}}(R, f)=\max \left\{|f(Z)|: Z \in \bar{G}_{R}\right\}$ and say that the function (11) belongs to the generalized convergence class if

$$
\int_{R_{0}}^{\infty} \frac{\alpha\left(\ln M_{\bar{G}}(R, f)\right)}{\beta(\ln R)} d \ln R<+\infty,
$$

where $\alpha \in L$ and $\beta \in L$. The following theorem is true.

Theorem 1. Suppose that the functions $\alpha$ and $\beta$ satisfy the assummptions of Theorem $A$ and $A_{k}=\max \left\{\left|a_{k_{1}, \ldots, k_{n}}\right|: k_{1}+\cdots+k_{n}=k\right\}$. In order that the function (11) belong to the generalized convergence class it is necessary and in the case when $\left|A_{k}\right| /\left|A_{k+1}\right| \nearrow+\infty$ as $k_{0} \leq k \rightarrow \infty$ it is sufficient that

$$
\sum_{k=0}^{\infty}(\alpha(k)-\alpha(k-1)) \beta_{1}\left(\frac{1}{k} \ln \frac{1}{\left|A_{k}\right|}\right)<+\infty, \quad \beta_{1}(x)=\int_{x}^{\infty} \frac{d t}{\beta(t)} .
$$

Proof. We use a method of A.A. Gol'dberg [5], applied for finding of formulas for the determination of the order and the type of the function $\ln M_{\bar{G}}(R, f)$.

We denote

$$
P_{k}\left(\xi_{1}, \ldots, \xi_{n}\right)=\sum_{k_{1}+\cdots+k_{n}=k} a_{k_{1}, \ldots, k_{n}} \xi_{1}^{k_{1}}, \ldots, \xi_{n}^{k_{n}}
$$

Then [5]

$$
\max \left\{M_{\bar{G}}\left(1, P_{k}\right) R^{k}: k>0\right\} \leq M_{\bar{G}}(R, f) \leq F_{1}(R)=: \sum_{k=1}^{\infty} M_{\bar{G}}\left(1, P_{k}\right) R^{k} .
$$

Since $\max \left\{M_{\bar{G}}\left(1, P_{k}\right) R^{k}: k>0\right\}=\mu\left(R, F_{1}\right)$ and the functions $\ln \mu\left(R, F_{1}\right)$ and $\ln F_{1}(R)$ belong to the same generalized convergence class, the relation (12) holds iff

$$
\int_{R_{0}}^{\infty} \frac{\alpha\left(\ln \mu\left(R, F_{1}\right)\right)}{\beta(\ln R)} d \ln R<+\infty
$$

Let

$$
F_{2}(R)=\sum_{k=1}^{\infty} B_{k} R^{k}, \quad B_{k}=: \max _{k_{1}+\cdots+k_{n}=k}\left(\left|a_{k_{1}, \ldots, k_{n}}\right| d_{\bar{G}}\left(k_{1}, \ldots, k_{n}\right)\right) .
$$

By Lemma 3 from [5] $B_{k} \leq M_{\bar{G}}\left(1, P_{k}\right) \leq B_{k}(k+1)^{n}$ and, therefore,

$$
\begin{gathered}
\ln \mu\left(R, F_{2}\right) \leq \ln \mu\left(R, F_{1}\right) \leq \max \left\{\ln B_{k}+n k+k \ln R: k>0\right\}= \\
=\max \left\{\ln B_{k}+k(\ln R+n): k>0\right\}=\ln \mu\left(R e^{n}, F_{2}\right) .
\end{gathered}
$$

Hence it follows that

$$
\int_{R_{0}}^{\infty} \frac{\alpha\left(\ln \mu\left(R, F_{2}\right)\right)}{\beta(\ln R)} d \ln R \leq \int_{R_{0}}^{\infty} \frac{\alpha\left(\ln \mu\left(R, F_{1}\right)\right)}{\beta(\ln R)} d \ln R \leq \int_{R_{0}}^{\infty} \frac{\alpha\left(\ln \mu\left(R e^{n}, F_{2}\right)\right)}{\beta(\ln R)} d \ln R
$$


and in view of the condition $\beta \in L^{0}$ the relation (15) is equivalent to the relation

$$
\int_{R_{0}}^{\infty} \frac{\alpha\left(\ln \mu\left(R, F_{2}\right)\right)}{\beta(\ln R)} d \ln R<+\infty .
$$

Since the closed domain $\bar{G}$ is bounded, contained in some polycylinder $Z_{L}=\left\{Z:\left|z_{j}\right| \leq L\right\}$. Therefore, $d_{\bar{G}}\left(k_{1}, \ldots, k_{n}\right)=d_{k}^{k}$, where $l \leq d_{k} \leq L$ and, thus,

$$
B_{k}=d_{k}^{k} \max _{k_{1}+\cdots+k_{n}=k}\left|a_{k_{1}, \ldots, k_{n}}\right|=A_{k} d_{k}^{k}
$$

and $\ln \mu\left(R, F_{2}\right)=\max \left\{\ln A_{k}+k \ln d_{k}+k \ln R\right\}$, that is

$$
\ln \mu\left(l R, F_{3}\right) \leq \ln \mu\left(R, F_{2}\right) \leq \ln \mu\left(L R, F_{3}\right),
$$

where $F_{3}(R)=\sum_{n=1}^{\infty} A_{k} R^{k}$. From (18) in view of the condition $\beta \in L^{0}$ it follows that the relation (17) and

$$
\int_{R_{0}}^{\infty} \frac{\alpha\left(\ln \mu\left(R, F_{3}\right)\right)}{\beta(\ln R)} d \ln R<+\infty .
$$

are equivalent. By Theorem $\mathrm{A}$ in order that inequality (19) hold, it is necessary and in the case $\left|A_{k}\right| /\left|A_{k+1}\right| \nearrow+\infty$ as $k_{0} \leq k \rightarrow \infty$ it is sufficient that (14) holds. Theorem 1 is proved.

3. Convergence $\Phi$-class. We say that the function (11) belongs to the convergence $\Phi$-class $(\Phi \in \Omega)$ if

$$
\int_{R_{0}}^{\infty} \frac{\Phi^{\prime}(\ln R) \ln M_{\bar{G}}(R, f)}{\Phi^{2}(\ln R)} d \ln R<+\infty .
$$

The results of the section are announced in [6] without the proof.

Theorem 2. Let the function $\Phi \in \Omega$ satisfy the condition (7), (9), and $\Phi^{\prime}(\sigma+O(1)) \asymp \Phi^{\prime}(\sigma)$ as $\sigma \rightarrow+\infty$. In order that (20) hold it is necessary and in the case when $\left|A_{k}\right| /\left|A_{k+1}\right| \nearrow+\infty$ as $k_{0} \leq k \rightarrow \infty$ it is sufficient that

$$
\sum_{k=k_{0}}^{\infty} \frac{1}{\Phi^{\prime}\left(\frac{1}{k} \ln \frac{1}{\left|A_{k}\right|}\right)}<+\infty
$$

Proof. In view of a result from [3] and the inequalities (14) the relation (20) holds if and only if

$$
\int_{R_{0}}^{\infty} \frac{\Phi^{\prime}(\ln R) \ln \mu\left(R, F_{1}\right)}{\Phi^{2}(\ln R)} d \ln R<+\infty .
$$

For the function (16) as above we have $\ln \mu\left(R, F_{2}\right) \leq \ln \mu\left(R, F_{1}\right) \leq \ln \mu\left(R e^{n}, F_{2}\right)$, whence

$$
\begin{gathered}
\int_{R_{0}}^{\infty} \frac{\Phi^{\prime}(\ln R) \ln \mu\left(R, F_{2}\right)}{\Phi^{2}(\ln R)} d \ln R \leq \int_{R_{0}}^{\infty} \frac{\Phi^{\prime}(\ln R) \ln \mu\left(R, F_{1}\right)}{\Phi^{2}(\ln R)} d \ln R \leq \\
\leq \int_{R_{0}}^{\infty} \frac{\Phi^{\prime}(\ln R) \ln \mu\left(R e^{n}, F_{2}\right)}{\Phi^{2}(\ln R)} d \ln R
\end{gathered}
$$


and according to the condition $\Phi^{\prime}(\sigma+O(1)) \asymp \Phi^{\prime}(\sigma)$ as $\sigma \rightarrow+\infty$ the relation (22) is equivalent to the condition

$$
\int_{R_{0}}^{\infty} \frac{\Phi^{\prime}(\ln R) \ln \mu\left(R, F_{2}\right)}{\Phi^{2}(\ln R)} d \ln R<+\infty
$$

For the function $F_{3}(R)=\sum_{n=1}^{\infty} A_{k} R^{k}$ we have (18) and, therefore, in view of the condition $\Phi^{\prime}(\sigma+O(1)) \asymp \Phi^{\prime}(\sigma)$ as $\sigma \rightarrow+\infty$ the relation (23) hold if and only if

$$
\int_{R_{0}}^{\infty} \frac{\Phi^{\prime}(\ln R) \ln \mu\left(R, F_{3}\right)}{\Phi^{2}(\ln R)} d \ln R<+\infty .
$$

By Theorem B in order that inequality (24) holds, it is necessary and in the case when $\left|A_{k}\right| /\left|A_{k+1}\right| \nearrow+\infty$ as $k_{0} \leq k \rightarrow \infty$ it is sufficient that (21) holds.

Choosing properly the function $\alpha$ and $\beta$ or $\Phi$ from Theorems 1 and 2 we obtain suitable corollaries. If we choose for example $\Phi(\sigma)=e^{\rho \sigma}, 0<\rho<+\infty$, then we obtain the following statement for an entire function of finite order.

Corollary 1. In order that $\int_{R_{0}}^{\infty} \frac{\ln M_{\bar{G}}(R, f)}{R^{\rho+1}} d R<+\infty$, it is necessary and in the case when $\left|A_{k}\right| /\left|A_{k+1}\right| \nearrow+\infty$ as $k_{0} \leq k \rightarrow \infty$ it is sufficient that $\sum_{k=k_{0}}^{\infty}\left|A_{k}\right|^{\rho / k}<+\infty$.

For an entire function of finite logarithmic order $p \in(1,+\infty)$ we choose $\Phi(\sigma)=\sigma^{p}$ for $\sigma \geq \sigma_{0}$.

Corollary 2. In order that $\int_{R_{0}}^{\infty} \frac{\ln M_{\bar{G}}(R, f)}{R \ln ^{p+1} R} d R<+\infty, p>1$, it is necessary and in the case when $\left|A_{k}\right| /\left|A_{k+1}\right| \nearrow+\infty$ as $k_{0} \leq k \rightarrow \infty$ it is sufficient that

$$
\sum_{k=k_{0}}^{\infty}\left(\frac{1}{k} \ln \frac{1}{\left|A_{k}\right|}\right)^{1-p}<+\infty .
$$

We wish to thank Professor O. B. Skaskiv for his useful suggestions.

\section{REFERENCES}

1. Sheremeta M.M. On two classes of positive functions and belonging to them of main characteristic of entire functions// Mat. Stud. - 2003. - V.19, №1. - P. 73-82.

2. Mulyva O.M. Convergence classes in the theory of Dirichlet series// Ukr. Mat. Zh. - 1999. - №3. - P. 35-39. (in Ukrainian)

3. Filevych P.V., Sheremeta M.M. On a convergence class for entire functions// Bull. Soc. Sci. Lettres Lodz, 53, Ser. Rech. Deform. - 2003. - V.40. - P. 5-16.

4. Mulyava O.M., Sheremeta M.M. On a convergence class for Dirichlet series// Bull. Soc. Sci. Lettres Lodz, 50, Ser. Rech. Deform. - 2000. - V.30. - P. 23-30.

5. Gol'dberg A.A. Elementary remarks on the formulas for the determination of the order and the type of entire funtions of several variables// Dokl. AN Arm. SSR. - 1959. - V.29, №4. - P. 145-151. (in Russian)

6. Mulyava O.M. On the belonging of entire functions of several variables to convergence classes// Modern stientific-methodical problems in higher school. Materials of International stientific-methodical Conference: NUFT, 2015. - P. 149-151. (in Ukrainian)

7. Skaskiv O.B., Tarnovetska O.Yu. On convergence classes for series, similar to Taylor-Dirichlet series// Bukovyn. mat. journ. - 2015. - V.3, №3-4. - P. 170-172. (in Ukrainian)

Ivan Franko National University of Lviv 